\title{
Posner Schlossman Syndrome, a case series study
}

\author{
Shams Mohammed Noman ${ }^{1 *}$, Umme Salma Akbar ${ }^{2}$ and Dr Tasmia Tahmid ${ }^{3}$ \\ ${ }^{1}$ Bangabandhu shekh mujib medical university, Bangladesh \\ ${ }^{2}$ Chittagong eye infirmary and training complex, Bangladesh \\ ${ }^{3}$ Cheveron eye hospital, Bangladesh
}

*Corresponding author: Dr Shams md Noman associate prof, Bangabondhu Shekh Mujib Medical University, Bangladesh.

\begin{abstract}
Background: The purpose of the current study is to describe clinical manifestations, management and its outcome of patients who were diagnosed as posner-schlossman syndrome (glaucomatocyclitic crisis) at the Glaucoma department Chittagong eye infirmary and training complex, Chittagong

Method: It is a hospital based observational case series study. Study period was from 1st july 2012-to 30th june2014.Unilateral ocular hypertension cases of unexplained origin referred to glaucoma clinic from outpatient department of CEITC were included in this study. Detail history taking and ocular examinations were done that included stitlampbiomicroscopy, applanationtonometry, gonioscopy,fundoscopy and visualfield analysis. Posner schlossman syndrome or Glaucomatocyclitic crisis (PSS) was diagnosed according to mentioned criteria. Management detail was recorded. Patients were followed up after one weak, 1 month,3 months of initial visit. Examination and investigation findings were documented as much as possible.

Result: A total numbers of 45 patients with PSS (posner schlossman syndrome) were included. For the better understanding the results, the total patients $(\mathrm{N}=45)$ were categorized into 2 age groups that are 20-49 and 50+. Percentage of patients into these groups are $51.1 \%(\mathrm{n}=23)$ and $48.9 \%(\mathrm{n}=22)$ respectively. In total 45 patients, 37 were male and 8 were female. $53.3 \%$ patients $(\mathrm{n}=24)$ presented with decreased visions, $15.6 \%$ patients $(\mathrm{n}=7)$ with both pain and occasional redness, $13.3 \%$ patients $(\mathrm{n}=6)$ with mild discomfort, $11.1 \%$ patients $(\mathrm{n}=5)$ with recurrent redness, $8.9 \%$ patients $(n=4)$ with occasional halos, $4.4 \%$ patients $(n=2)$ with occasional eye ache and $2.2 \%$ patient $(n=1)$ with loss of vision. Mean IOP of affected eyes was $30 \mathrm{~mm} \mathrm{hg}$ before treatment and $15 \mathrm{~mm} \mathrm{hg}$ after treatment. Angles were open in all cases. At presentation $81.2 \%$ patients (n-37) presented with glaucomatous optic disc C:D ratio (0.8-1):1. 77.77\% patients(n=35) needed flurometholone, 4.44\% cases(n=2) needed dexamethasone and $4.44 \%$ patients $(n=2)$ needed prednisolone acetate topical eye drop to control inflammation. 13.33\% patients ( $n=6)$ were not treated with any steroid as they resolved spontaneously. $91 \%$ patients needed anti glaucoma medications $9 \%$ needed filtration surgery with Ologen implantation to control IOP.

Conclusion: Early diagnosis with meticulous examinations and investigations are needed to manage a PSS case. As it is an elusive disease, proper explanation of the disorder to the patient to get good compliance from them is necessary to achieve good medical treatment outcome. Good filtration surgery with Ologen implantation is still successful to control IOP. But regular careful monitoring to the patient is mandatory to perfectly manage and prevent the irreversible visual consequences of recurrence of the disease.
\end{abstract}

Key Words: PSS (Posner schlossman syndrome); GCC (Glaucomatocyclitic crisis); Inflammation; Open angle; Trabeculectomy

\section{Introduction}

Posner schlossman syndrome is an uncommon form of secondary open angle glaucoma. It is a condition with self-limiting recurrent episodes of markedly elevated intraocular pressure
(IOP) with mild idiopathic anterior chamber inflammation [1,2]. In 1948, Posner and Schlossmann published a series of 9 patients and described the features of this syndrome as unilateral, recurrent 
episodes of mild non-granulomatous uveitis; with elevated IOP during the acute attack, which persists for a few hours to several weeks; corneal edema with a few keratic precipitates; heterochromia with anisocoria; open angles; normal visual fields; and normal optic discs. In between the attacks, the IOP, outflow facility and provocative tests were within normal limits [1]. Since this original description, other cases attributed to Posner schlossman syndrome have been found to deviate from these criteria [3]. Additional features that are now recognized are, this condition affects individuals aged 20-50 years; both eyes may be involved at different times but rarely simultaneously $[4,5,6]$. The rise of IOP is out of proportion to the severity of the uveitis, and this rise in IOP precedes the less identifiable inflammatory reaction, often by several days. A significant number of patients with PSS develop glaucoma overtime [4]. Filtration surgery is needed to prevent damage from high IOP with frequent attacks. Anti-glaucoma agents and surgery do not prevent recurrences of PSS. In most of the cases, it remains undiagnosed or sometimes over-diagnosed. Few symptoms and signs which are self-remitting, respond to steroid but the recurrent attack of high IOPs leads to glaucomatous damage. Loss of vision from glaucoma is an important factor in the management of these patients. The purpose of the current study is to describe clinical manifestations and management of patients who were diagnosed as Posner Schlossman syndrome at the Glaucoma Department of Chittagong Eye Infirmary and Training Complex, Chittagong, Bangladesh.

\section{Method}

This is a hospital based observational case series study. Study period: 1st July 2009to 30th June2011. In this period, old cases of previously diagnosed PSS and newly diagnosed cases were included. New cases were followed up for at least 3 visits (1 week,1month, 3 months and/or 6 months). The following data were recorded: age of the patient at onset, gender, presenting complaints, mean IOP, visual acuity, grade of inflammation before and after treatment, optic disc appearance, medications, glaucoma surgery if needed and post-operative follow up. Diagnosis of Posner Schlossman syndrome was made by the following criteria: mild anterior

Table 1: Age Group.

Table 1: Age Group.
\begin{tabular}{|c|c|c|}
\hline Age Group & Frequency & Percent \\
\hline $20-49$ & 23 & 51.1 \\
\hline $50+$ & 22 & 48.9 \\
\hline Total & 45 & 100 \\
\hline Mean 48.0, SD +15.33 & & \\
\hline
\end{tabular}

Table 2: Sex and Age Group Distribution.

\begin{tabular}{|c|c|c|c|}
\hline \multirow{2}{*}{ Sex } & \multicolumn{2}{|c|}{ Age Group } & \multirow{2}{*}{ Total } \\
\cline { 2 - 4 } & \multicolumn{2}{|c|}{$\mathbf{2 0 - 4 9}$} & $\mathbf{5 0 +}$ \\
\hline Male & 19 & 18 & 37 \\
\hline Female & 4 & 4 & 8 \\
\hline
\end{tabular}

chamber inflammation with small non-pigmented kpS, open angle and absence of posterior synechiae. There was never any occurrence vitreous activity and cystoid macula edema. The attacks usually resolved by 2 to 3 weeks, sometimes spontaneously, and sometimes it was recurrent. Between attacks, the eyes were normal. IOPs were measured using Goldmann applanationtonometry. Patients were diagnosed as developing glaucoma from attacks of PSS as follows:

- $\quad$ The IOP in the affected eye was $>21 \mathrm{mmHg}$.

- The affected eye had glaucomatous optic disc and/or visual field changes.

Details of anterior segment with slit lamp biomicroscopywere examined. Optic disc examination was done by indirect ophthalmoscope with 90D and 78D lens. Optic disc criteria for glaucoma included [6-9]:

\section{- $\quad$ Cup/disc ratio greater than 0.7}

- Notching or pathognomic change of neuroretinal rim or focal pit.

- Narrowest remaining neuroretinal rim of 0.1-disc diameter or less

- $\quad$ Asymmetry in cup/disc ratio of more than 0.2

- $\quad$ Progressive thinning of the neuroretinal rim

All patients were reviewed by a single consultant. Data analysis was done by SPSS V-13.

\section{Results}

A total number of 45 patients with PSS were included during the study period. The mean age of the patients was 48 years Table 1. The age of the patients ranged from 20 years to 50 years and above. For better understanding of the results, the total patients ( $n=45)$ were categorized into 2 age-groups: 20 to $49 \mathrm{yrs}$ and $>50 y$ rs. Percentage of patients in these groups are $51.1 \%(n=23)$ and $48.9 \%(n=22)$ respectively. Of the total 45 patients,37 were male and 8 were female. Numbers of male and female were almost equal in both age groups (Table 1\&2). 
$53.3 \%$ patients $(n=24)$ presented with decreased vision; both pain and occasional redness were complaints of $15.6 \%$ patients $(n=7) ; 13.3 \%$ patients $(n=6)$ presented with mild discomfort. $11.1 \%$ patients $(n=5)$ presented with recurrent redness, and $8.9 \%$ patients( $(n=4)$ with occasional halos. $4.4 \%$ patients $(n=2)$ and $2.2 \%$ patient $(\mathrm{n}=1)$ presented with occasional redness and loss of vision respectively Table 3. Mean IOP of affected eye was $30 \mathrm{mmHg}$ and $15 \mathrm{mmHg}$ before and after treatment respectively (Table 3\&4).

Table 3: Presenting Complaints.

\begin{tabular}{|c|c|c|}
\hline Complaints & Responses N=56 & Percent of Cases \\
\hline Pain & 7 & $15.60 \%$ \\
\hline Decrease Vision & 24 & $53.30 \%$ \\
\hline Occasional Redness & 7 & $15.60 \%$ \\
\hline Occasional Haloes & 4 & $8.90 \%$ \\
\hline Occasional eye ache & 2 & $4.40 \%$ \\
\hline Recurrent redness & 5 & $11.10 \%$ \\
\hline Discomfort & 6 & $13.30 \%$ \\
\hline Loss of vision & 1 & $2.20 \%$ \\
\hline
\end{tabular}

Table 4:

\begin{tabular}{|c|c|}
\hline \multicolumn{2}{|c|}{ Mean IOP of affected eye } \\
\hline Before Treatment & After Treatment \\
\hline 30 & 15 \\
\hline
\end{tabular}

Of the 45 patients, $42.2 \%$ (n=19) had VA of $6 / 6-6 / 18,20 \%(n=9)$ had VA less than $6 / 18-6 / 60$ and $37 \%$ patients(n=17) presented with VA less than $6 / 60$ before treatment. After treatment $80 \%$ patients(n=36) had VA of $6 / 6-6 / 18,13.3 \%(n=6)$ had VA $<6 / 18$ $6 / 60$ and $6.7 \%$ patients $(n=3)$ had $V A<6 / 60$ (Tables $5 \& 6$ ).

Table 5: Visual acuity of affected eye before Treatment.

\begin{tabular}{|c|c|c|}
\hline VA & N & Percent \\
\hline $6 / 6-6 / 18$ & 19 & 42.2 \\
\hline$<6 / 18-6 / 60$ & 9 & 20 \\
\hline$<6 / 60$ & 17 & 37.8 \\
\hline Total & 45 & 100 \\
\hline
\end{tabular}

Table 6: Visual acuity of affected eye after Treatment.

\begin{tabular}{|c|c|c|}
\hline VA & N & Percent \\
\hline $6 / 6-6 / 18$ & 36 & 80 \\
\hline$<6 / 18-6 / 60$ & 6 & 13.3 \\
\hline$<6 / 60$ & 3 & 6.7 \\
\hline Total & 45 & 100 \\
\hline
\end{tabular}

Table 7: Inflammation before treatment.

\begin{tabular}{|c|c|c|}
\hline Inflammation & Frequency & Percent \\
\hline+ & 41 & 80.39 \\
\hline++ & 4 & 7.84 \\
\hline KP & 6 & 11.76 \\
\hline
\end{tabular}

Mild anterior chamber reaction (that is cell 1+) was found in 80.39\% $(n=41)$ of patients and 7.84\% $(n=4)$ patients had cells $2+$ in anterior chamber. At presentation $81.2 \%$ patients $(n=37)$ presented with glaucomatous optic disc (C:D ratio 0.8-1); $13.4 \%$ presented $(n=6)$ with C:D ratio 0.6 to 0.7 . Only $4.4 \%$ patients $(n=2)$ had C:D ratio between 0.3-0.4 (Table 7\&8).

Table 8: $C D$ ratio in affected eye.

\begin{tabular}{|c|c|c|}
\hline & Frequency & Percent \\
\hline $0.3: 1$ & 1 & 2.2 \\
\hline $0.4: 1$ & 1 & 2.2 \\
\hline $0.6: 1$ & 3 & 6.7 \\
\hline $0.7: 1$ & 3 & 6.7 \\
\hline $0.8: 1$ & 15 & 33.3 \\
\hline $0.9: 1$ & 18 & 40 \\
\hline $1: 01$ & 4 & 8.9 \\
\hline Total & 45 & 100 \\
\hline
\end{tabular}

Intraocular pressure was controlled by medical treatment in $91 \%$ cases. $9 \%$ cases needed surgery. $31.1 \%(n=14)$ patients were treated with one anti-glaucoma medication (timolol maleate $0.5 \%)$ and $68.9 \%(n=31)$ patients needed combination therapy (Timolol maleate and brimonidinetartrat $0.1 \%$ ).77.77\% of patients $(n=35)$ needed fluoromethalone, $4.44 \%$ of cases $(n=2)$ needed dexamethasone and $4.44 \%$ patients $(n=2)$ were treated with prednisolone to control inflammation. $13.33 \%$ patients $(n=6)$ were not treated with any steroid as they spontaneously resolved (Figures 1).

\section{Medical Treatment}

(Tables $9 \& 10$ and Figures 2,3,4,5)

Table 9: Anti-glaucoma medication.

\begin{tabular}{|c|c|c|}
\hline Medicine & $\mathbf{N}$ & $\mathbf{\%}$ \\
\hline Timolol & 14 & $31.10 \%$ \\
\hline Timolol + Brimonidine & 31 & $68.90 \%$ \\
\hline Total & 45 & $100 \%$ \\
\hline
\end{tabular}

Table 10: Steroid use.

\begin{tabular}{|c|c|c|}
\hline Medicine & $\mathbf{N}$ & $\mathbf{\%}$ \\
\hline Fluorometholone & 35 & $77.77 \%$ \\
\hline Dexamethasone & 2 & $4.44 \%$ \\
\hline Prednisolone & 2 & $4.44 \%$ \\
\hline No steroid & 6 & $13.33 \%$ \\
\hline Total & 45 & $99.98 \%$ \\
\hline
\end{tabular}

\section{Discussion}

Posner Schlossman syndrome is an uncommon form of secondary open angle glaucoma. Diagnosis of PSS is difficult, and in most cases, it is misdiagnosed as it mimics other ocular conditions [7]. Here we reported 45 cases of Posner Schlossman syndrome within two years. This indicates it is not so uncommon 
in Bangladesh. Posner Schlossman syndrome typically affects individuals aged $20-50$ years [8,9]. In our study $51.1 \%$ patients were in the 20 to 49 yrs age group and $48.9 \%$ patients belonged to the above 50 years old age group. Thus, older patients should also be carefully monitored. Males were predominant in both age groups. $53.3 \%$ of patients came into hospital with the complaints of decrease vision. As PSS is self-remitting with minimal symptoms, repeated attacks are not noticed in most of the patients [10]. When vision decreased, patients then visited the hospital. Pain and redness were the second commonest symptoms (15.6\%). Some patients visited with typical features of PSS like discomfort, occasional halos, recurrent redness etc. Intraocular pressures in PSS are usually high ranging from 40 to $60 \mathrm{mmHg}[11,12]$. In our study, the mean IOP before treatment was $30 \mathrm{mmHg}$ and after treatment IOP was controlled. Before treatment $37.8 \%$ patients visited with $\mathrm{VA}<6 / 60$ and $42.2 \%$ with $6 / 6-6 / 18$. After treatment $80 \%$ patients retain their vision within $6 / 6-6 / 18$. Blurred vision in acute attacks is due to high IOP which induces corneal edema. After treatment $19.7 \%$ patients reported $V A<6 / 18$ visual acuity due to glaucomatous damage of the disc. $81.2 \%$ of patients presented with glaucomatous optic disc in which the cup disc ratio is 0.8 to 0.9 . Thus, PSS is not always benign as previous thought to be described by Posner. The repeated attacks of high IOP causes permanent damage to the disc. In this observational case series study, every patient was asked to return earlier if they had an attack, but all patients were not interested to come for every attack due to the self-limiting and short-lived nature of the disease, so it is difficult to determine the duration of elevated IOP during each episode and the numbers of attacks. So, in this study we could not explore the possibility of duration of the attack and number of attacks as being factors responsible for causing glaucoma in these patients.
The aim of medical treatment is to control inflammation and IOP elevation. The favored initial treatment is a combined regimen of a topical nonsteroidal anti-inflammatory drug (NSAID) and an antiglaucoma drug (topical beta-blockers and/or carbonic anhydrase inhibitors). In our study, Fluorometholone was used to control inflammation in $77.77 \%$ patients. Timololmaleate was used as first line treatment for IOP control. Combination therapy was necessary in $68.9 \%$ patients. Topical carbonic anhydrase inhibitors were not given as it is costly and not available in all places in Bangladesh. Topical NSAIDs like Bromofenac was used in 11\% patients. Surgical intervention was indicated when increase frequency of attacks and high fluctuation of IOP which may result in progressive optic nerve damage. $9 \%$ of cases needed filtration surgery (trabeculectomy) due to IOP not being controlled by only medical treatment and also when patient had nerve damage. Post-operatively, IOP remained controlled after 1 week, 1 month and 3 months. However, again in a short period case series study, there may have been missed episodes of follow up. Both Medical and surgical treatment does not prevent recurrences, but glaucoma filtration surgery is able to control high rise of IOP even during the cyclitic attacks [5]. It may decrease the numbers of attacks [5]. Good functioning filtering bleb may decrease the inflammatory cells from the anterior chamber and ultimately prevent the bleb failure. Hence filtration surgery should be advised when any glaucomatous change has occurred. Because patients may miss follow ups, high spikes of IOP in every attack are not always noticed. The underlying cause of PSS still remains a debate. Cytomegalovirus seems to be the most likely infectious cause [13]. New insights into possible infectious etiologies of PSS may lead to the development of effective treatment strategies to prevent recurrence for this elusive disease. In our study we did not attempt to investigate causative factors (Figures 6).

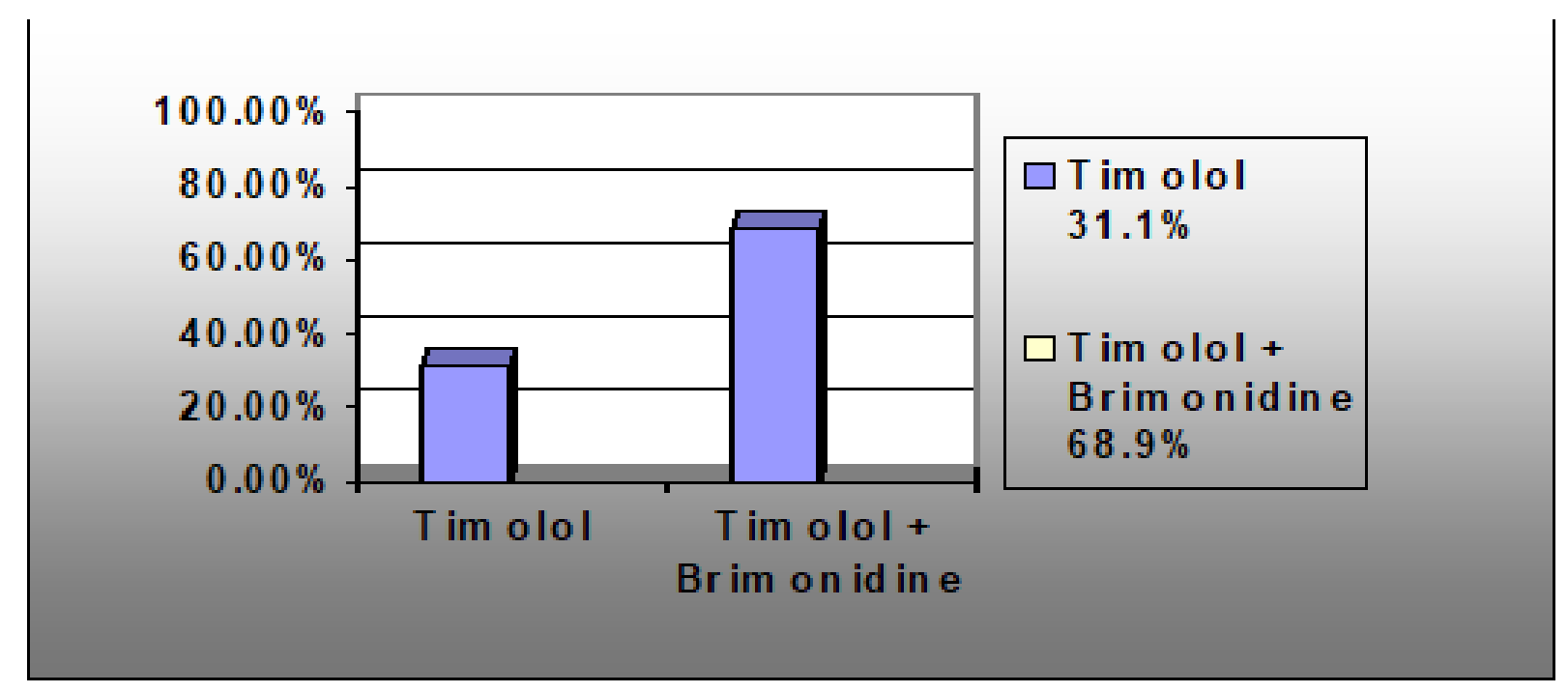

Figure 1: Bar chart for different anti-glaucoma medications. 


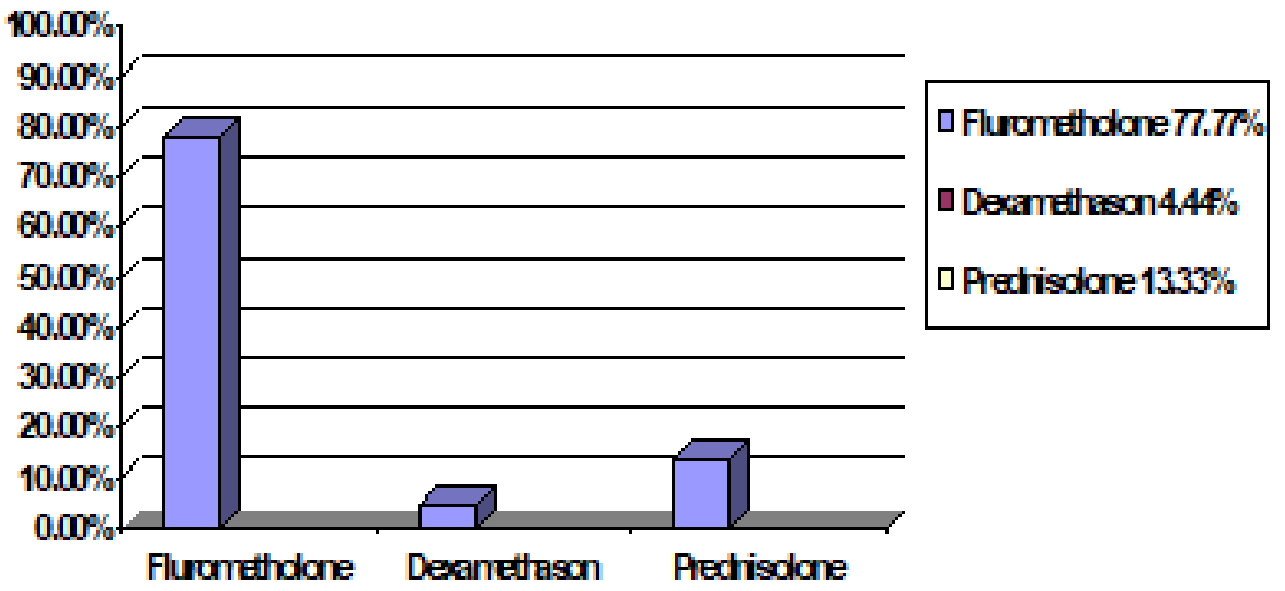

Figure 2: Bar chart for steroid used.

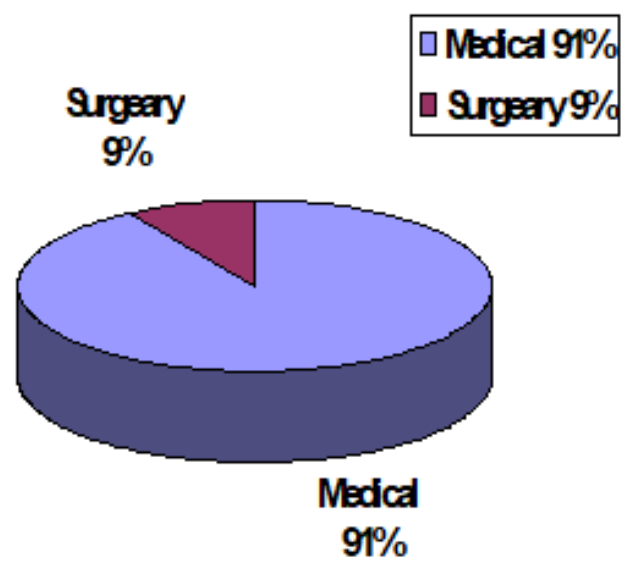

Figure 3: Pie chart for management options.
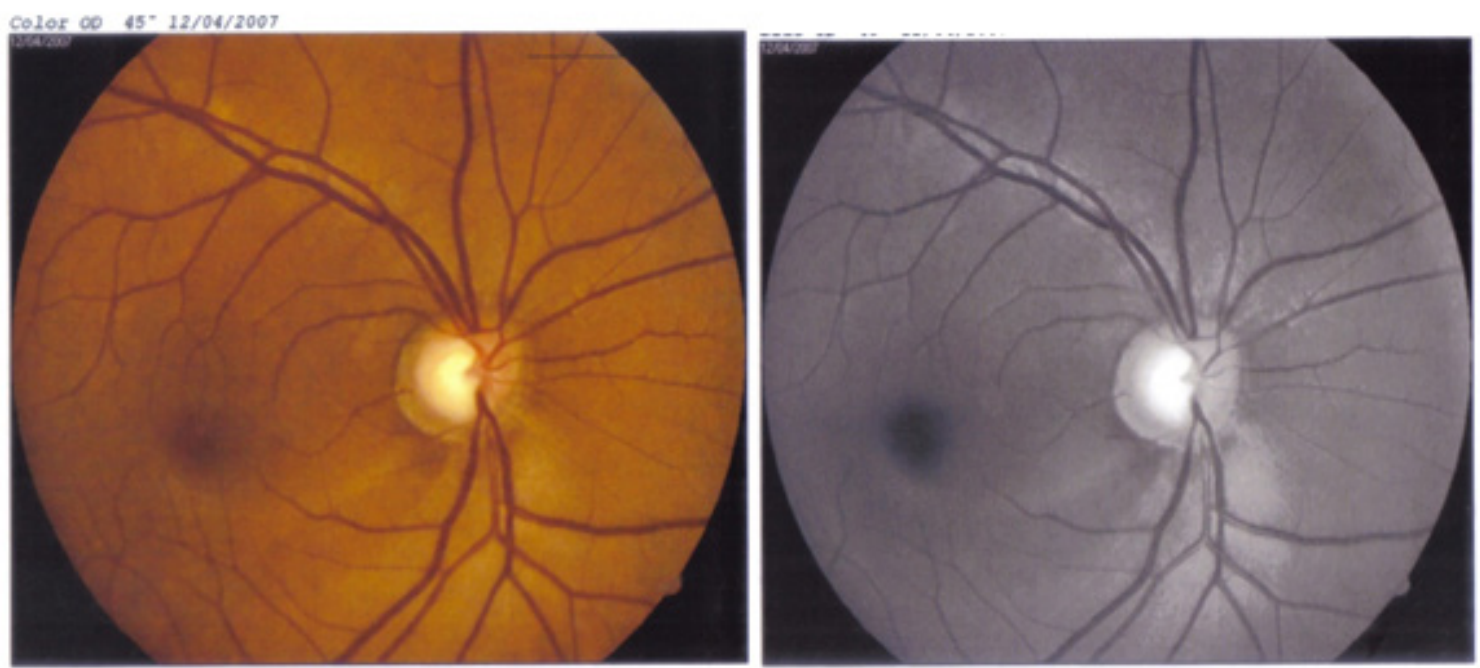

Figure 4: 


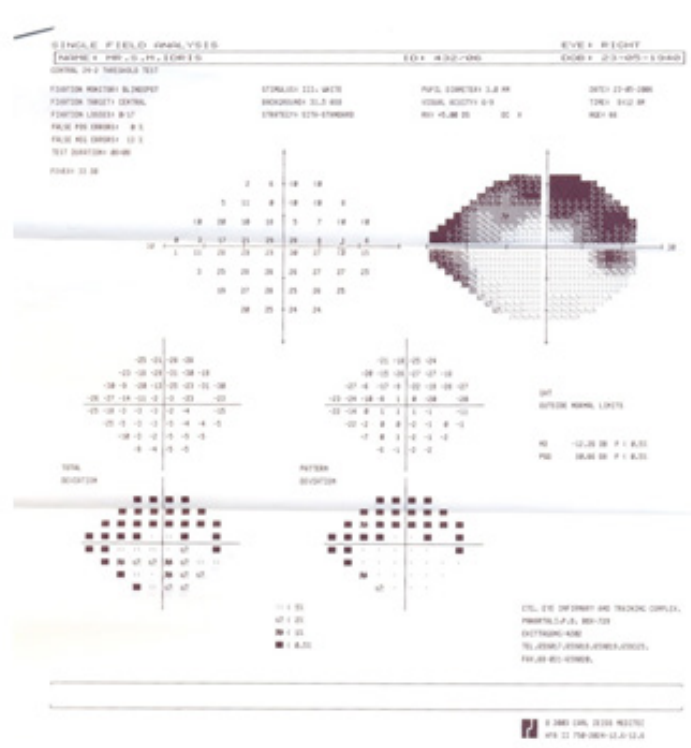

Figure 5: Glaucomatous disc with nerve fiber loss with corresponding visual field defect.

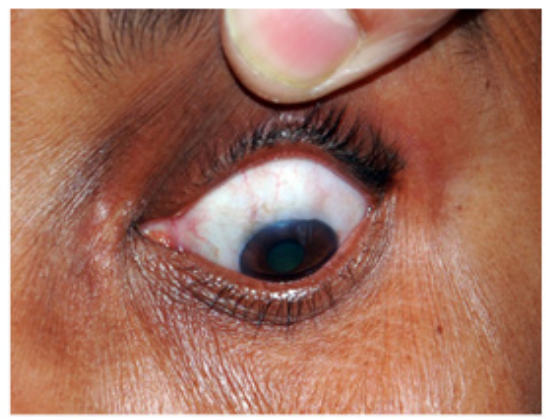

Figure 6: Good filtering bleb after trabeculectomy.

\section{Conclusion}

Posner schlossman syndrome is an uncommon but not rare disease. Early diagnosis with meticulous examination and investigationsare needed. Proper explanation of the disorder to the patient to get good compliance is necessary to achieve good treatment outcomes as it is an elusive disease (since the course of disease is not well understood). Good filtration surgery is still successful to control IOP, but regular careful monitoring of the patient is mandatory to perfectly manage and prevent the irreversible visual consequences of recurrent attacks.

\section{Acknowledgement}

Chittagong eye infirmary and training complex

\section{Conflict of Interest}

None.

\section{References}

1. Posner A, Schlossman A (1948) Syndrome of unilateral recurrent attacks of glaucoma with cyclitic symptoms. Arch Ophthalmol 39(4): 517-535.
2. Raitta C, Vannas A (1977) Glaucomatocyclitic crisis. Arch Ophthalmol 95: 608-612.

3. Kass MA, Becker B, Kolker AE (1973) Glaucomatocyclitic crisis and primary open-angle glaucoma [case report]. Am J Ophthalmol 75(4): 668-673.

4. Hung PT, Chang JM (1974) Treatment of glaucomatocyclitic crises. Am J Ophthalmol 77(2): 169-172.

5. Caprioli J (1990) Automated perimetry in glaucoma. In: Walsh TJ (Eds.), Visual Fields: Examination and terpretation. San Francisco: American Academy of Ophthalmology 3: 71-106.

6. Sommer A, Pollack I, Maumenee AE (1979) Optic disc parameters and onset of glaucomatous field loss II. Static screening criteria. Arch Ophthalmol 97(8): 1449-1454.

7. Pederson JE, Anderson DR (1980) The mode of progressive disc cupping in ocular hypertension and glaucoma. Arch Ophthalmol 98(3): 490-495.

8. Zeyen TG, Caprioli J (1993) Progression of disc and field damage in early glaucoma. Arch Ophthalmol 111(1): 62-65.

9. Yamada N, Mills RP, Leen MM, Emond MJ, Reynolds AC, et al. (1997) Probability maps of sequential Glaucoma-scope images help identify significant changes. J Glaucoma 6(5): 279-287.

10. Weinreb RN (1987) Adjusting the dose of 5-fluorouracil after filtration surgery to minimize side effects. Ophthalmology 94(5): 564-570. 
11. Stavrou P, Murray PI (1999) Does trabeculectomy influence the course of uveitis. Ocul Immunol Inflamm 7(2): 103-108.

12. Hill RA, Nguyen QH, Baerveldt G, Forster DJ, Minckler DS, et al. (1993) Trabeculectomy and Molteno implantation for glaucomas associated with uveitis. Ophthalmology 100(6): 903-908.
13. Hana L, Takusagawa, Yao Liu, Janey L Wiggs (2011) Infectious theories Theories of posner-Schlossman Syndrome. Int Ophthalmol Clin 51(4): 105-15. 